and

$$
p_{2 i-1}(\mathrm{~B}) \cdot p_{2 i}(\mathrm{~A})=p_{i}(1)
$$

therefore

$$
p_{i}(0)=p_{i}(1)
$$

where $p_{i}(0)$ and $p_{i}(1)$ are probabilities of zeros and ones in the $i$-th place of the sequence $\left\{Y_{k}\right\}$.

The procedure above described may be used for production of binary random numbers by automatic digital computers. In this case the manual switch $S$ must be replaced by an electronic switch, of course.

Warszawa, Poland

Z. PAWLAK

\title{
Numerical Solution of the Schroedinger Equation for Central Fields
}

A fast program has been written for the ILLIAC to integrate the radial SCHROEDINGER equation

$$
\begin{gathered}
u^{\prime \prime}(r)-\left[l(l+1) / r^{2}+2 V(r)-E\right] u=0 \\
\text { boundary conditions: } u(0)=0 \quad u(r) \text { bounded }
\end{gathered}
$$

for any well-behaved potential $V(r)$. More generally the program can integrate any linear second-order differential equation which can be put in the form

$$
u^{\prime \prime}(r)-g(r) u(r)=q(r)
$$

with $q(r)$ vanishing at zero and infinity, $r^{2} g(r)$ bounded at zero, and $g(r)$ bounded for large $r$ [1].

A distinctive feature of the program is the use of the Noumerov [2], [3] method for the integration. This is faster than techniques (such as the RunGEKUTTA) which depend on making an estimate of one or more forward points and improving this by an iteration scheme. Here there is no iteration, but the error in the "estimate" of each forward point is of eighth order in the step-size $h$, so that the truncation error may still be kept small. The essence of the method is the elimination of all odd powers of $h$ from the TAYLOR expansion about any point by working with three points instead of two, followed by a change of dependent variable which removes the $h^{4}$-term. The calculation of a forward point to order $h^{8}$ thus requires the value of the dependent variable at the six preceding points.

For an equation in the form (2), the required new dependent variable is

$$
y=u-\left(h^{2} / 12\right)(g u+q) .
$$

The prescription for calculating forward points is

$$
y_{n+1}=2 y_{n}-y_{n-1}+h^{2} \frac{g_{n} y_{n}+q_{n}}{1-\frac{1}{12} h^{2} g_{n}}-\frac{1}{240} \delta^{6} y_{n},
$$


where the sixth central difference in the last term is given, to order $h^{8}$, by

$$
\delta^{6} y_{n}=3 \delta^{6} y_{n-2}-2 \delta^{6} y_{n-3} \text {. }
$$

This prescription can be used as soon as $y$ is known at the first six points.

To get this far, i.e., to start the integration, an iteration method is used: The difference equation (4) without the last term is solved by successive approximations at the first six points, guessing a value of $y_{0}$ and improving this guess by applying the condition (seventh order approximation)

$$
\delta^{6} y_{2}=\delta^{6} y_{3}
$$

For the inhomogeneous equation $(q \neq 0)$, where the normalization is not arbitrary, the starting value $u_{1}=u(h)$ is found by another iteration scheme: With an estimated $u_{1}$, the equation is integrated out to some large $r$, where the inhomogeneity $q$ has become negligible. Application of the condition that $u$ be small and monotonically decreasing leads to an improved estimate of $u_{1}$. This scheme, which seldom requires more than three iterations, is practical because of the speed of integration. (ILLIAC takes less than 40 milliseconds per $h$-step.)

In its present form, the program can-

-find eigenvalues $E$ (permitted negative energies)

-print out values of the wave functions $u=u_{E}^{(r)}$ at desired values of $r$, and find the nodes and extrema

-evaluate matrix elements $\int u_{E_{i}} u_{E_{j}}$ (with ILLIAC's electrostatic memory of $2^{10}$ words, at 40 bits per word, there is enough space to integrate several wave-functions simultaneously)

-solve self-consistent field problems (Schroedinger equation and PoIsson equation solved simultaneously).

A "guide" to the program has been prepared, containing a detailed description of the mathematical method and instructions for use of the existing tapes, and is available in mimeographed form at the University of Illinois Digital Computer Laboratory.

The authors are indebted to Professors John Blatt and J. N. Snyder for helpful suggestions.

\section{R. A. RubENSTEIN \\ MARJORIE Huse \\ STEFan Machlup}

Dept. of Physics

University of Illinois

Urbana, Illinois

1. If $q(r)$ is not identically zero, i.e., for the inhomogeneous equation, there is the additional restriction that $r^{2} g(r)$ be bounded everywhere.

2. G. W. PRATT, JR., "Wave functions and energy levels for $\mathrm{Cu}^{+}$as found by the Slater approximations to the Hartree-Fock equations," Phys. Rev., 88, 1952, p. 1217.

3. B. Noumerov, Monthly Notices Roy. Astron. Soc., 84, 1924, p. 592. 